\title{
Surface-mediated photoalignment of organic/inorganic nanohybrids
}

\author{
Takahiro SEKI, Haruhiko FUKUMOTO, Mitsuo HARA, Nobuhiro KAWATSUKI* and Shusaku NAGANo
}

Department of Molecular Design and Engineering, Graduate School of Engineering, Nagoya University, Chikusa, Nagoya 464-860

*Department of Materials Science and Chemistry, Graduate School of Engineering, University of Hyogo,

2167, Shosha, Himeji 671-2201

\begin{abstract}
Light irradiation provides significant tools for material structuring and processing. In this article, the photo-orientation processes of the organic/inorganic hybrid materials recently achieved in our laboratory are briefly introduced. Irradiation of linearly polarized light to a polymer film containing a photoisomerizabke azobenzene or photocrosslinkable cinnamoyl unit leads to the induction of anisotropic molecular orientations on the surface. This molecular anisotropic surface structure can be transferred to a controlled orientation of nanostructures of organic/inorganic hybrid films at macroscopic scales. Two classes of lyotropic/silica systems have been applied to the photo-orientation procedures, i.e., ordinary surfactant systems and chromonic ones. These processes can be regarded as the extended applications of the so-called command surface systems, which photochemically regulate the molecular orientations of liquid crystals.
\end{abstract}

(C) 2008 The Ceramic Society of J apan. All rights reserved.

Key-words: Organic/Inorganic hybrids, Photoalignment, Azobenzene, Cinnamate, Liquid crystals, Choromonics

[Received November 5, 2007; Accepted December 11, 2007]

1. Introduction

In biological systems, varieties of molecular processes are consorted with each other to perform highly sophisticated functions, where mineral components are produced and oriented under strong influences of organic molecular membranes (biomineralization). ${ }^{1)}$ Also in artificial mimicking systems, structures and morphologies of inorganic materials are modified by the chemical and supramolecular architecture of the organic molecular assembly. ${ }^{1)}$ Light irradiation provides significant tools for materials processing, however, no attempts have been made to date to achieve on-demand orientational controls of stiff inorganic materials in response to light. For soft or fluid organic matters, on the other hand, light-induced modifications are frequently achieved with incorporation of photochromic dyes. Use of linearly polarized light (LPL) leads to an anisotropic alignment. When polymer films or Langmuir-Blodgett (LB) films possessing a photochromic unit (mostly azobenzene $(\mathrm{Az})$ ) are exposed to LPL, the excited chromophore undergoes a reorientation to a non-excitable direction, namely to the orthogonal direction.

In this review article, the photo-orientation processes of the organic/inorganic hybrid materials recently achieved in our laboratory are introduced. Organic molecules-templated silicates are intriguing candidate for photo-orientation because such hybrid materials are synthesized via sol-gel polymerization starting form a fluid lyotropic liquid crystalline precursor. Two classes of lyotropic/silica systems have been subjected to the photo-orientation, i.e., ordinary surfactant systems and chromonic ones. The former is now widely known for synthesis of mesoporous silica, and the latter is less investigated but should be important for providing optical devices.

2. Induction of molecular alignment by light

The most widely employed photoreactive unit for photo- alignment of liquid crystalline materials is azobenzene $e^{2), 3)}$ (Fig. 1, left). Research on Az-containing polymeric films has exploded during the past few decades, and it is continuing to be a very active area of materials chemistry. This unit undergoes the $E$ (trans)- to $Z$ (cis)-form photoisomerization under UV light (e.g., $365 \mathrm{~nm}$ line of $\mathrm{Hg}$ lamp) irradiation, and reversible process occurs with light of longer wavelength (e.g., $436 \mathrm{~nm}$ line of $\mathrm{Hg}$ lamp). ${ }^{4)}$ The cis-totrans back reaction also proceeds via thermal process. An alternative strategy for the photoalignment of molecular orientatin is to apply photocrosslinking $([2+2]$ photocyclization) of two cynnamate groups ${ }^{5), 6)}$ (Fig. 1, right). This reaction is thermally irreversible. Thus, the resultant structural anisotropy becomes robust, which allows the sol-gel process achieved under heated conditions. This situation favors the application to photoalignment of organic/silica

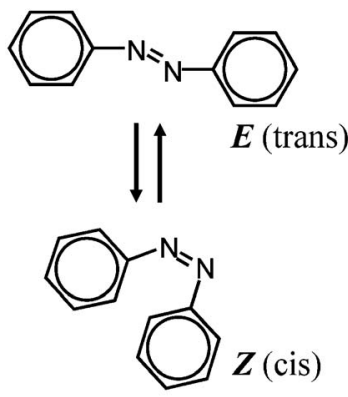

photoisomerization

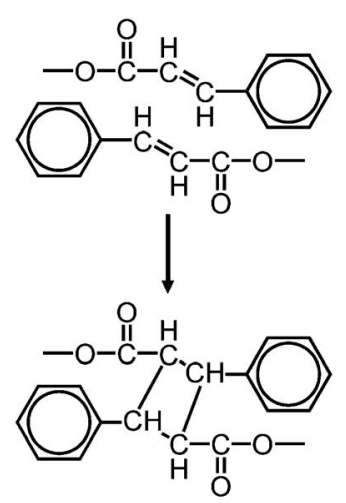

$(2+2)$ photocyclization
Fig. 1. Typical photoreacting units for photoalingment of liquid crystalline materials. 
hybrids.

The photoalignment process involves angular selective photoexcitation of the above photoreactive units in a polymer or molecular assembly films. Use of linearly polarized light (LPL) leads to an anisotropic alignment. When a layer material possessing a photoractive unit are exposed to LPL, the excited chromophore undergoes a reorientation to a nonexcitable direction, namely to the orthogonal direction. ${ }^{7)-14)}$ This effect is called the photoinduced optical anisotropy or Weigert effect. This photoinduced anisotropy can be transferred to the orientation of liquid crystals, $\left.\left.{ }^{7,}, 8\right), 10\right)$ dye aggregates ${ }^{15)}$ and polymer chains. ${ }^{16)}$ Most typical orientational transfer is attained from azobenzene monolayer to nematic liquid crystals. Due to the large amplification of the information possessed by the surface, the system is called 'command surface' (Fig. 2). 17),18) The photoalignment of organic/inorganic hybrid structures can be regarded as the extension of this surface aligning principle.

3. Photo-alignment of surfactant-templated nanostructured silica

Mesoporous (nanoporous) materials ${ }^{19)}$ having compartmentalized nano-spaces such as MCM-4120),21) and FSM-

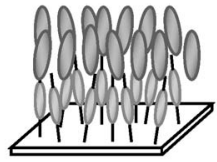

Homeotropic

Homeotropic

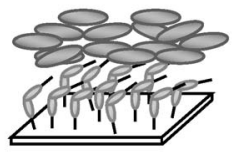

Homogeneous

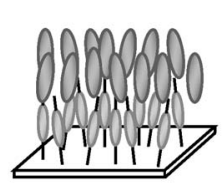

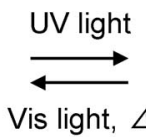

Vis light, $\Delta$
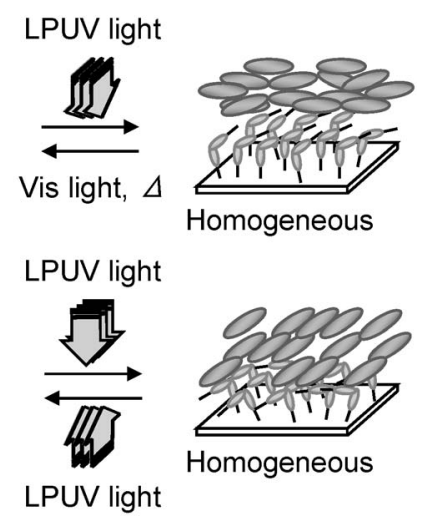

Fig. 2. Schematic illustrations of the surface-mediated photocontrol of thermotropic liquid crystal alignment triggered by photoresponsive moieties placed on a substrate surface.
$16^{22), 23)}$ which are obtained by templating of organic surfactant assemblies have been attracting increasing attentions in catalysts, ${ }^{24)}$ optical materials, ${ }^{25), 26)}$ separations, molds of polymers ${ }^{27)}$ and fabrication of noble metal wires (Fig. 3).28),29) In general, mesoporous materials are obtained as powders. The mesochannels may be highly directional at local levels, however, they are randomized at larger scale levels, which would impair their potential technological applications. The macroscopically aligned mesostructured silica is available by application of strong magnetic fields, reactant flows ${ }^{30)}$ and capillary flow with an elastic micromold for microcontact printing in electric fields. ${ }^{31}$ ) Thin films are also available via spincasting ${ }^{32)}$ or nucleation at interfaces. ${ }^{33), 34)}$ In thin films, a macro-scale orientation of mesochannels can be attained by deposition on a rubbed surface $^{35)}$ or an LB film ${ }^{36)}$ of polyimide. Nevertheless, such mechanical procedures do not benefit for fine fabrications including micropatterning. This section describes photoorientation procedures for the mesostructured surfactant/ silica hybrids. In fact, two types of photo-orientation are attempted, namely 1) via multi-step transfers among heterointerfaces starting from an azobnezene monolayer, and 2) via single-step transfer from a crosslinkable liquid crystalline polymer film as the alignment layer.

\subsection{Approaches and photoalignment layers}

The first demonstration of photoalignment of mesostructured silica hybrid has been performed by Kawashima et al. ${ }^{37), 38)}$ In this approach, the photoorientation of the $\mathrm{Az}$ monolayer (6Azn-PVA, poly (vinyl alcohol) possessing an azobenzene side chain) is first achieved by irradiation of LPL. Here, the long axis of azobenzene is oriented preferentially orthogonal to the polarization plane of LPL. The in-plane anisotropy of the 6Azn-PVA monolayer is transferred to a photoorientated spincast film of a poly (di- $n$ hexylsilane) (PDHS), ${ }^{16)}$ and successively deposition of mesoporous silica film is performed (Fig. 4, left). The condensation of tetraethoxysilane (TEOS) to silica network is achieved under acid conditions. The thickness of resulting silica hybrids ranges some hundred nanometers. This 6Az10PVA/PDMS system has a drawback from practical viewpoints as follows. i) The process is complicated and demands handling skills. ii) The orientation of composite film does not survive temperatures above $40^{\circ} \mathrm{C}$; therefore the structure of channels lacks mechanical stability due to insufficient crosslinking of the siloxane condensation.

To overcome these problems, Fukumoto et al. ${ }^{39)-41)}$ have proposed a new versatile photo-alignment method using a photo-crosslinkable side-chain-type polymer with

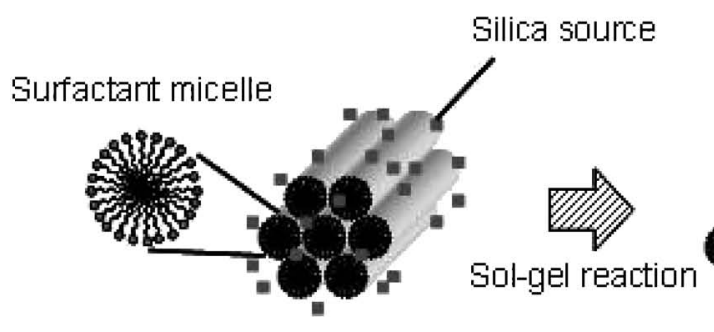

Micellar rods (template)
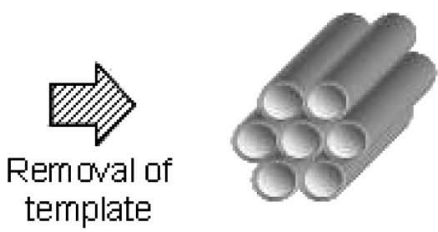

Mesoporous silica

Fig. 3. Synthetic scheme of mesoporous silica via templating of liquid crystalline surfactant rod-like micelles. 


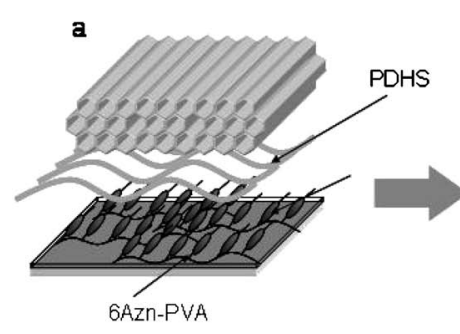

b

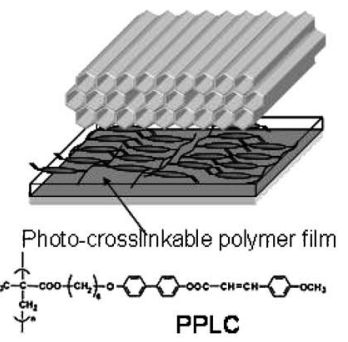

Fig. 4. Photoalignment of silica mesochannels by photoreactive polymer layers based on the photoisomerization (a) and photociclyzation (b).

LC nature (PPLC), poly6-(4-(4'-methoxycinnamoyloxy) biphenyloxy) hexylmethacrylate developed by Kawatsuki et al., ${ }^{42), 43)}$ in which the mesogenic biphenyl group is combined with a cinnamoyl terminal (Fig. 4, right). In many types of photo-crosslinkable polymers, an optical anisotropy of angular selective $[2+2]$ photodimerization between two cinnamoyl groups undergoes by exposure to LPL. ${ }^{44), 45)}$ For PPLC under investigation, the photoinduced orientational anisotropy of the film is largely amplified through selfassembling nature of liquid crystal by annealing. Another advantage to use the photo-crosslinkable polymer as the PPLC is that the resultant photochemical crosslinking firmly fixes the anisotropic molecular orientation. This feature allows facile performances of siloxane condensation at higher temperatures without damages of the alignment polymer film.

\subsection{Structural characterizations of mesochannels}

Clear evidence for the formation of uniaxially aligned mesochannels is obtained by transmission electron microscopy (TEM) for the photoaligning layer of 6Az10-PVA/ PDHS. ${ }^{38)}$ The two TEM photographs in Fig. 5 depict the cross-sectional structure of the as-synthesized surfactant (cetyltrimethylammonoium (CTAC))-templated nanostructured silica hybrid film observed in the two directions orthogonal to each other. Figure 5a shows the image when the film is sliced parallel to the direction of the actinic polarized light. The hexagonal structure of the mesochannels is clearly visualized through overall thickness of the film. On the other hand, when the film is sliced perpendicular to the direction of the actinic polarized light, the lines with a periodicity of 3-4 nm running parallel to the substrate are observed (Fig. 5b). The cylindrical structure of the mesochannels is fully stretched straight in the film. This TEM image obviously visualizes the hetero-interface regions composed of the $\mathrm{Si}$ wafer (the dark region at the bottom), Az monolayer ( $2 \mathrm{~nm}$ thickness, not clearly shown), The PDHS film (40-60 nm thickness, brighter region) and deposited mesostructured silica with parallel stripes. The orientational information possessed by the $\mathrm{Az}$ monolayer is transferred to the alignment of PDHS of ca. $50 \mathrm{~nm}$ thickness and then to mesostructured channels of ca. 400-500 nm thickness. Thus, great amplifications are attained.

The uniaxially aligned honeycomb mesochannel structure attained in macroscopic areas can be justified from X-ray diffraction (XRD) patterns. An example is shown in Fig. 6 for CTAC-templated mesostructured channels aligned by
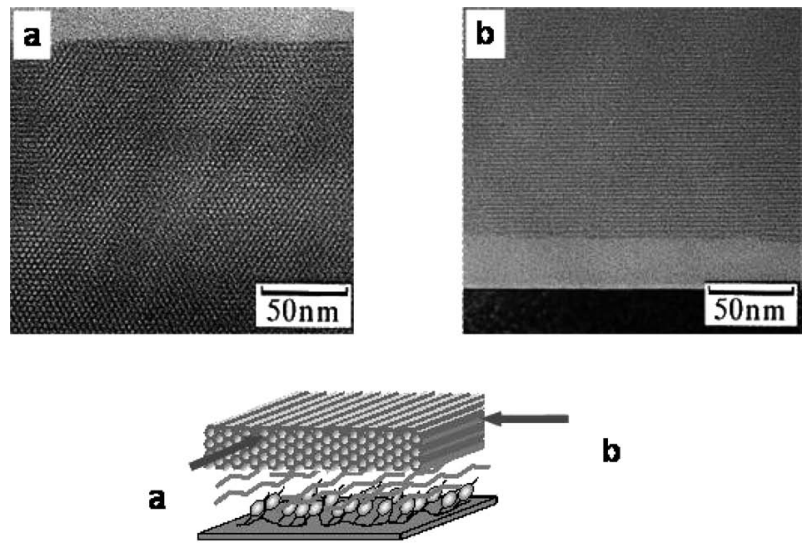

Fig. 5. TEM images of photo-aligned silica mesochannels. $a$ and $b$ indicate the directions of observation.
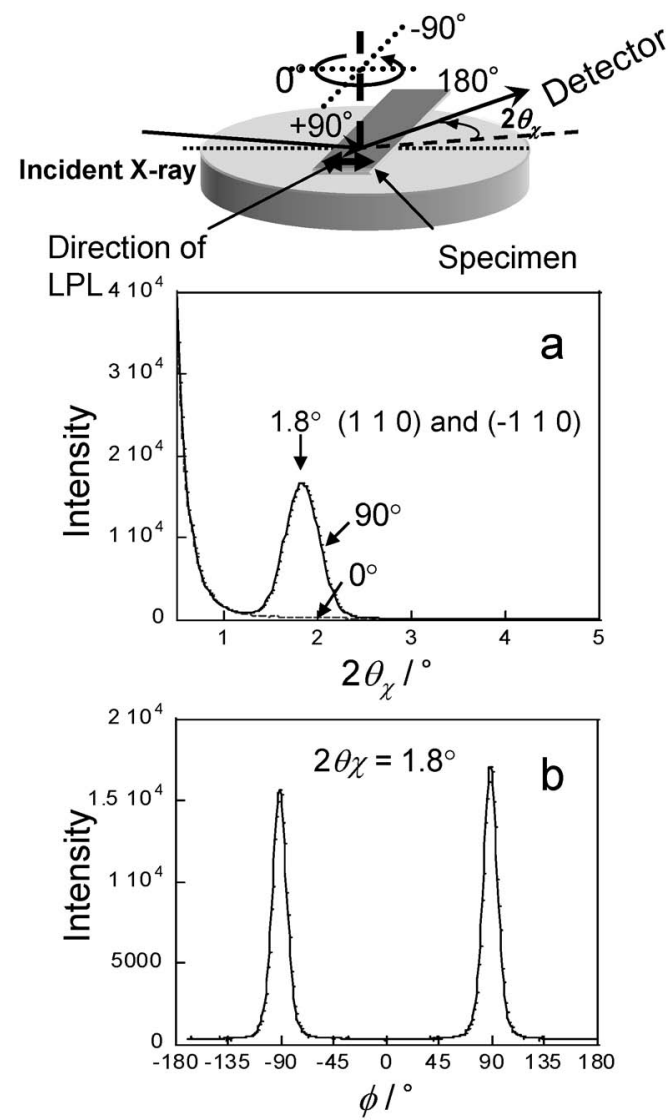

Fig. 6. In-plane XRD measurements to evaluate the photoalignment of mesochannels.

PPLC polymer film. The polarization direction of the LPUV light irradiated to the film coincides with parallel direction of incident X-ray at $\phi=0^{\circ}$. Fig. 6b shows $\phi-2 \theta_{\chi}$ XRD scan profiles of the as-synthesized mesostructured CTAC/silica composite thin film. At $\phi=0^{\circ}, 2 \theta_{\chi}$ XRD signal is not observed. When the sample is rotated to $\phi=+90^{\circ}$, strong diffractions at $2 \theta_{\chi}=1.8^{\circ}$ and $3.4-3.8^{\circ}$ assignable to $(-110)$ and (110) lattice plane perpendicular to the substrate, respectively, are observed (a). These results indicate that the 
mesochannels are photo-aligned perpendicular to the polarized direction of the irradiated linearly polarized light. Next, in-plane rotation $(\phi)$ of the silica thin film is conducted with retention of $2 \theta_{\chi}=1.8^{\circ}(\mathrm{b})$. In the $-180^{\circ}$ to $+180^{\circ}$ rotation, two obvious diffraction peaks with very narrow directional distribution are observed at $\phi=-90^{\circ}$ and $+90^{\circ}$ (b), indicating that the photo-oriented mesochannels have a preferential orientation perpendicular to the polarized direction of the LPL.

Mesoporous silica can be obtained after removal of the organic surfactant template from the composite. The surfactant molecules are generally removed by calcination by heating up to high temperatures exceeding $573 \mathrm{~K}$. Here, photo-decomposition (photocalcination) by vacuum UV light achievable at room temperature is applied according to a modified method of Hozumi et al. ${ }^{46)}$ It is expected that the distortion of the mesoporous structure in this method is more suppressed than that obtained via decomposition by heating. The complete removal of the organic template surfactant can be confirmed by Fourier transform infrared spectroscopy for a photoaligned composite film synthesized on a silicon wafer. Before the photo-decomposition of organic components, the symmetric and asymmetric bands of $\mathrm{CH}_{2}$ and $\mathrm{CH}_{3}$ stretching are observed in the range of $2840-2970 \mathrm{~cm}^{-1}$. On the other hand, the Si-O stretching band around $1070 \mathrm{~cm}^{-1}$ is essentially unchanged, indicative of the retention of siloxane network structure. Utilizing these photoaligned nanospaces, preparation of nanofibers via radical polymerization has been achieved. ${ }^{47)}$

Also the dip-coating method has been found to be applicable. $^{41)}$ In Fig. 7, in-plane XRD profiles of the CTAC/silica composite films prepared by the dip-coating are depicted. In the measurements, the direction of incident X-ray at $\phi=0^{\circ}$ coincides with the parallel direction of the dip-coat (lifting) direction. LPL is irradiated on the PPLC film in parallel $(/ /)$ and perpendicular $(\perp)$ to the dip-coating direction. In the same manners, a scan profile of in-plane rotation $(\phi)$ is obtained at $2 \theta_{\chi}=1.8^{\circ}$. In the case that the polarized direction of the irradiated LPL is set parallel (//) to the lifting direction, two sharp diffraction peaks are observed at $\phi=-90$ and $+90^{\circ}$ (b). In contrast, when the polarized direction is set perpendicular $(\perp)$ to the lifting direction, the diffractions are observed at $\phi=0^{\circ}$ and $\pm 180^{\circ}$. These facts clearly indicate that the mesochannels are aligned to the polarized direction of the LPL. It is stressed here that the channel orientation is predominantly controlled by the direction of photoaligning polymer layer and not by the lifting direction. The orientation degree of mesochannels is dependent on the thickness of the silica thin film. The thinnest film (thickness: $40 \mathrm{~nm}$ ) has the highest order of orientation of the mesochannels. The more effective photo-alignment can be achieved for the thinner film. These facts unequivocally indicate that the surface-mediated transcription is attained from the molecular orientation of the PPLC film to the surfactant rod-like aggregates.

\subsection{Photopatterning}

Micropatterned illumination of LPL to the PPLC film is achieved, and the morphology of the resulting silica film is observed by optical microscopy (Fig. 8). ${ }^{39), 41)}$ The PPLC film is irradiated with the LPL through a photomask and annealed. In the irradiated areas, linear cracks evolve, whose direction runs perpendicular to the polarized direction of the

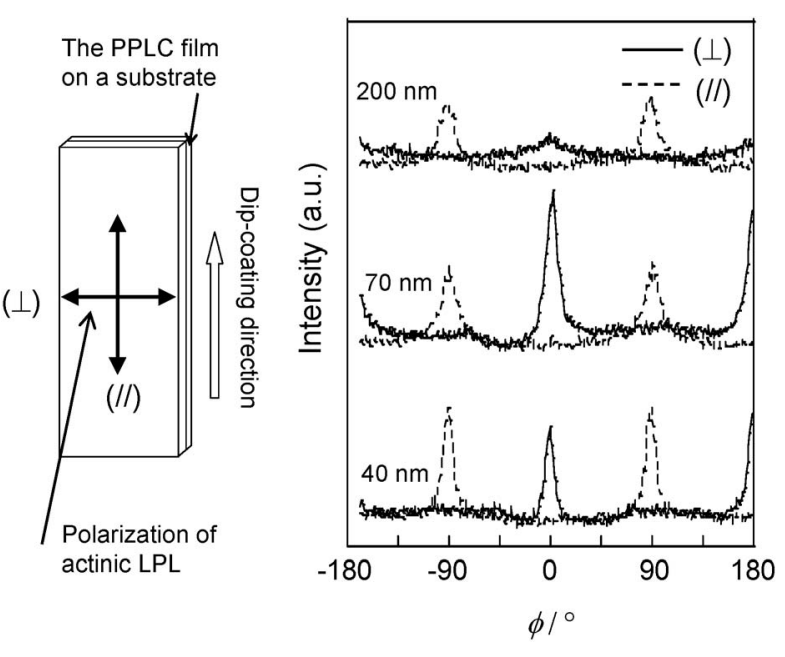

Fig. 7. In-plane XRD data of photoaligned messochannels prepared by dipping method.
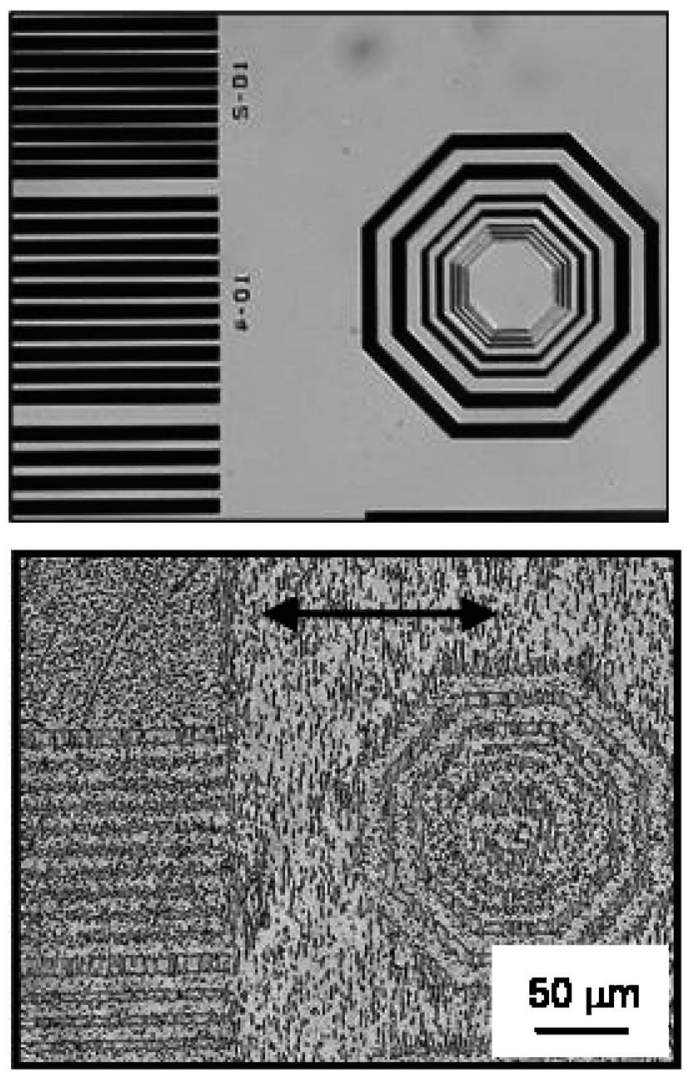

Fig. 8. Patterned photoalignment of messochannels using a photomask. Photomask (upper) and the obtained silica film (lower).

LPL. In contrast, grain morphologies are seen in the unilluminated areas. These morphological features strongly suggest that local alignments of mesochannels are attained in the geometry of the photomask. The patterning resolution in this experiment is ca. $10 \mu \mathrm{m}$.

4. Chromonics/silica nanohybrids

Vast numbers of studies have been undertaken to fabricate 
<smiles>COc1cc(N=Nc2cc(S(=O)(=O)O[Na])cc3cc(S(=O)(=O)O[Na])cc(O)c23)c(C)cc1N=Nc1c(O)cc2cc(Nc3ccccc3)ccc2c1O</smiles>

B67<smiles>NCCOCCO</smiles>

\section{AEE}

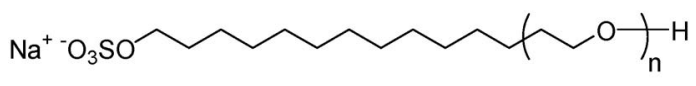

\section{Emal20C}

Fig. 9. Chemical structures of chromonic azo dye and additives in the TEOS sol solution.

mesoporous silica materials ${ }^{19)-23)}$ using various surfactanttemplated nanostructures. To date, almost all of the templates employed for these strategies are limited to 'typical' surfactant aggregates of low-molecular-mass compound $\mathrm{s}^{20)-23)}$ or amphiphilic block copolymers ${ }^{48)}$ that exhibit the lyotropic liquid crystal nature. In this section, a new type of lyotropic templating system of chromonic dye aggregates (chromonic liquid crystals) is indicated. The chromonic LLCs are composed of disc-like or plank-like aromatic dye molecules with hydrophilic units at the peripheries, and they can spontaneously self-assemble via $\pi-\pi$ stacking interaction and stack face to face to form columnar structures in aqueous solutions. $\left.{ }^{49)}-57\right)$

This section introduces the first demonstration of the fixation of columnar structure of the chromonic LLC by formation of silica networks. As a typical example for the chromonic LLC material, C.I. direct blue 67 (B67) is employed here (Fig. 9). An anionic surfactant Emal20E is also added, which stabilizes the nematic columnar phase. The immobilization of the columnar structure is successfully attained when an appropriate mediating molecule, 2-(2aminoethoxy) ethanol (AEE in Fig. 9), is added to the sol-gel process. ${ }^{58)}$ The significant role of mediator molecules ${ }^{59)-61)}$ in the structural stabilization of LLC materials is emphasized.

\subsection{Synthesis and characterization}

B67/silica hybrid films are prepared from a mixture of a dye solution and a sol solution. The nanostructured B67/silica hybrid films are prepared on glass substrates by the spin coating or dip coating. The films are dried overnight. The film thickness is ca. $100 \mathrm{~nm}$.

Figure 10a shows UV-Vis absorption spectra of the B67 solution (solid line) and the B67/silica hybrid films in the absence (dashed line) and presence (dotted line) of AEE measured at room temperature. In the aqueous solution, the spectrum of B67 dye exhibits a sharp peak with the absorp-
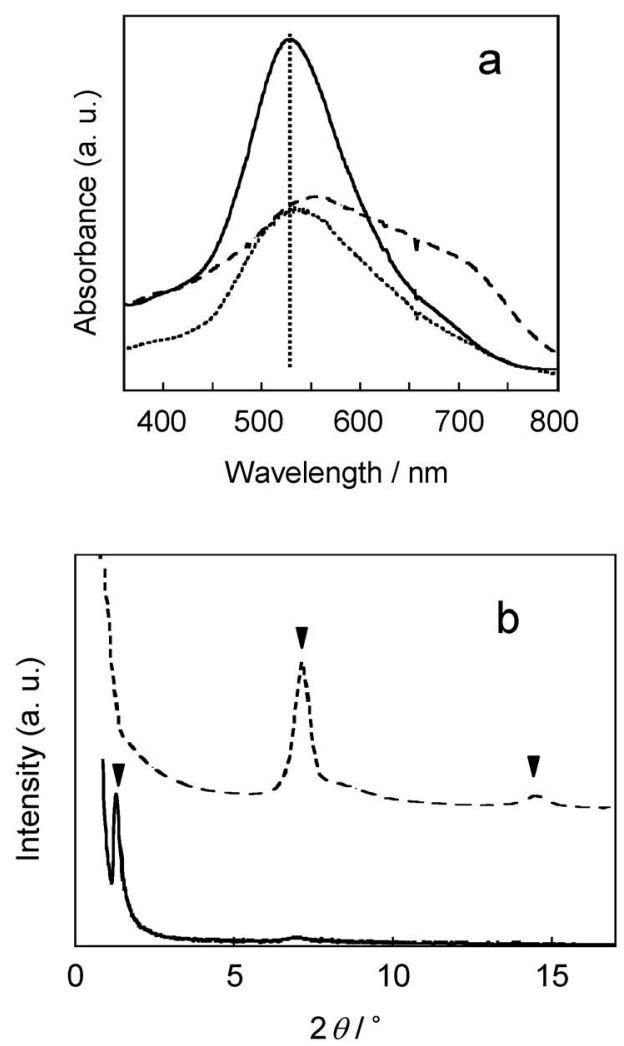

Fig. 10. UV-Vis spectra of chromonic silica sol solution (solid line) and the spin-coated B67/silica hybrid films without (dashed line) and with (dotted line) AEE (a), and XRD patterns of the spincoated B67/silica hybrid films with (solid line) and without (dashed line) $\operatorname{AEE}(b)$.

tion maximum $\left(\lambda_{\max }\right)$ at $530 \mathrm{~nm}$. The position of $\lambda_{\max }$ and the spectral feature indicate that B67 adopts an H-type aggregated state, implying the formation of B67-columnar structure. ${ }^{51)}$ On the other hand, the spincast hybrid film without AEE exhibits a broadened absorption band with the $\lambda_{\max }$ at $566 \mathrm{~nm}$ accompanied by an appearance of a shoulder at longer wavelengths around $700 \mathrm{~nm}$, suggesting a disruption of $\mathrm{H}$-aggregated columnar structure leading to multifarious stacking states of B67. In contrast, UV-Vis absorption spectrum of the silica hybrid film containing AEE give $\lambda_{\text {max }}$ at $532 \mathrm{~nm}$, and the spectral feature are almost the same as that of the B67 aqueous solution. These facts suggest that $\mathrm{H}$-like aggregate, and thus the columnar structure in the B67 solution can be retained in the hybrid film when AEE is present.

To obtain structural features of the hybrid film, the XRD measurement is performed (Fig. 10b). Contrasting results are obtained in the cases in the presence (solid line) and absence (dashed line) of AEE. For the film without AEE, clear diffraction peaks are observed at $7.3^{\circ}$ and $14.7^{\circ}$ for the hybrid film. These peaks are attributed to the (100) and (200) diffractions of an ordered lamellar structure with a spacing period $(d)$ of $1.32 \mathrm{~nm}$. On the other hand, a clear peak is observed at $1.3^{\circ}(d=6.78 \mathrm{~nm})$ in the hybrid film containing AEE. In this way, the addition of relatively small amount of AEE (a molar ratio of 0.5 of the total) induces the obvious structural change of the hybrid film. Judging from the knowledge of $\mathrm{H}$-aggregate formation from the 


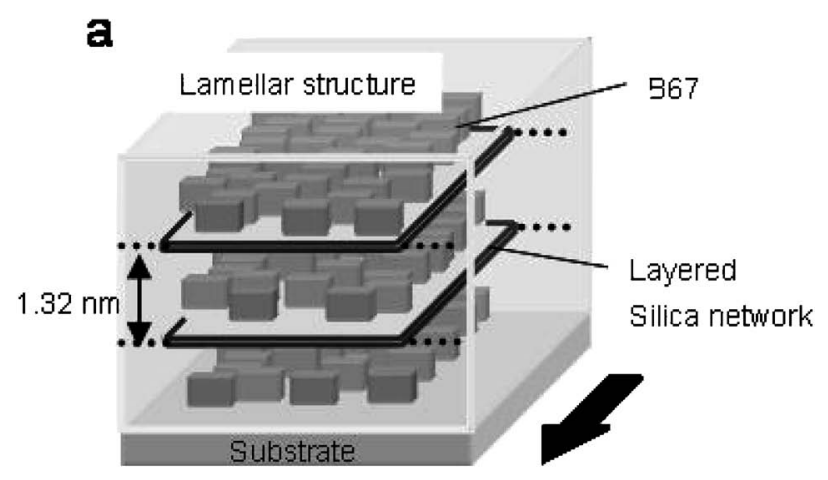

Lifting direction

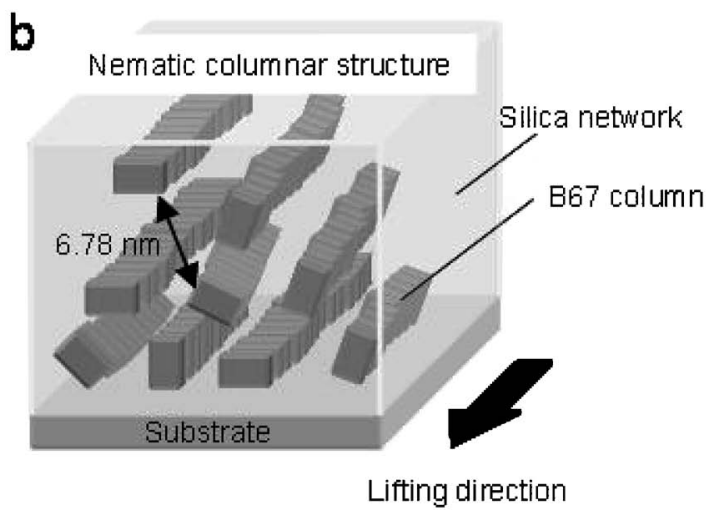

Fig. 11. Structural models of B67/silica hybrid films without (a) and with (b) AEE. The arrow indicates the lifting direction in the film forming process.

spectroscopic data and a reasonable coincidence with X-ray data by Ruslim et al., ${ }^{51)}$ this spacing should indicate the average distance between the columns of B67 aggregates. Figure 11 displays schematic illustrations of the structure of $\mathrm{B} 67 /$ silica nanohybrids in the absence (a) and presence (b) of AEE.

The addition of AEE brings about the stabilization of the columnar structure of B67 in the silica network. Most probably, AEE behaves as a mediator between the B67-columnar aggregates and silica network. It has been known that the organic/inorganic interfacial boundaries are varied by neutralization balance of electrostatic charge at the organic/ inorganic interfaces. The nanostructures of the hybrid materials strongly depend on the interfacial charge balance. ${ }^{60), 61)}$ Thus, the neutralization of charged surfaces at the organic/inorganic interface is important for the retention of nanostructures of the hybrid materials. In the present case, both the B67 columns with sulfonic acid unit and silica networks have the negative charged surface. The electric repulsion in the interface should destabilize the columnar structure in the high concentrated state, which can lead to the transformation to the lamellar structure when AEE is absent. AEE can neutralize the B67/silica interfaces in which the amine unit and ethylene oxide unit of AEE strongly interacts with the sulfonic acid units on the B67 columns and the silica network, respectively.

\subsection{Macroscopic orientation by flow}

When the dipping method is applied, a macroscopic in-plane optical anisotropy is attained by the lifting procedure. ${ }^{58)}$ The dichroic ratio $(D)$ defined as $A_{\perp} / A_{/ /}$, where $A_{\perp}$ and $A_{/ /}$are the absorbance at $\lambda_{\text {max }}$ taken in perpendicular and parallel to the polarized probing beam, respectively, is 1.5. The B67 molecules preferentially aligned orthogonal to the lifting direction, namely the direction of the column being parallel to this direction (see Fig. 11b). Thus far, the large scale alignment of chromonic liquid crystals is known to be performed by shear fields. ${ }^{56), 57)}$ The dipping method can be an alternative method for macroscopic alignment of chrominic liquid crystalline state state, which should be of technical significance. It is worth mentioning that the lamellar structured nanohybrid obtained without AEE also possesses macroscopic optical anisotropy (Fig. 11a), although the layer structure of the dye is separated by the silica layer. This fact suggests that the transformation (distortion) from the columnar to lamellar structure occurs after the flow alignment of the columns is attained in the course of drying. ${ }^{58)}$

\subsection{Photoalignment on PPLC Layer}

Photoalignment of the B67/silica columnar nanohybrid is attempted on the PPLC film. The chromonic liquid crystal of B67 is mixed with silica precursor (TEOS) together with an anionic surfactant of Emal20C and AEE. The hybrid film is prepared on the photo-oriented PPLC film by dipping into the precursor sol solution. After drying at ambient atmosphere overnight, hybrid films in deep purple color are obtained. The polarized UV-Vis absorption spectroscopy and in-plane grazing angle XRD measurements both indicate that the columnar structure is aligned uniaxially in the in-plane direction at a macroscopic level. Here the direction of the columns is aligned orthogonal to the polarization plane of the actinic LPL. In this case again, similarly to that of the surfactant template, the orienting power by the photoalignment layer of PPLC surpasses the flow effect of the dipping process. This situation allows effective photopattened alignment of the columnar structure of B67/silica hybrid as shown in Fig. 12. Work in this regard is now in progress and details on the photoalignment of the $\mathrm{B} 67$ chromonic/silica nanohybrid will be reported shortly. ${ }^{62)}$

\section{Conclusion}

This article overviewed the first examples to photoalign organic/inorganic nanohybrids achieved by our group. Among many choices of external stimuli to induce molecular orientations, light is particularly fascinating. Light approaches materials in non-contacting manners with high addressing accuracy. Also, the process does not require laborious and high-cost procedures. These advantageous features may lead to creations of new functional silica materials, particularly in fabrication of patterned devices. The photoalignment of organic/inorganic hybrids is just at the starting point of research. It is anticipated that further developments in the photoalignment of organic/inorganic hybrids will find many useful opportunities to exploit new functional materials and devices.

Acknowledgement We thank D. Matsunaga at Nippon Kayaku Co. and Dr. T. Tamaki at AIST for kindly providing B67 and Emal20C. We also thank T. Hikage for the technical 


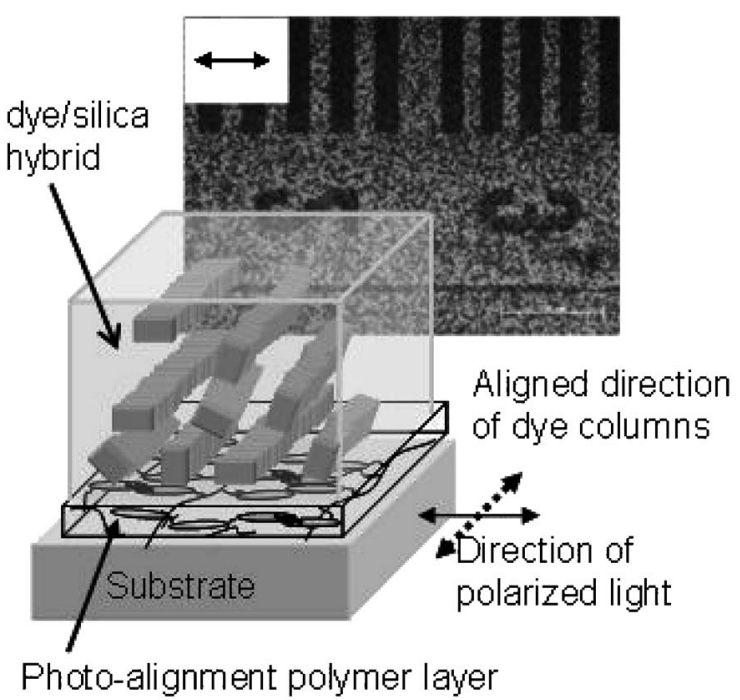

Fig. 12. Structural model of the B67/silica hybrid films on the PPLC photoalignment film and a polarized optical microscopic image obtained via micropatterned LPL irradiation.

assistance in the $\mathrm{X}$-ray measurements. This work was supported by a Grant-in-Aid for Science Research in a Priority Area "New Frontiers in Photochromism (No. 471)" and "Super-hierarchical Structures (No. 446)," and for Scientific Research A (16205019) and B (19350056) from the Ministry of Education, Culture, Sports, Science and Technology (MEXT), Japan.

\section{References}

1) S. Mann, Biomimetic Materials Chemistry, $\mathrm{VCH}$, Weinheim (1996).

2) G. S. Hartley, Nature, 140, 281 (1937).

3) G. S. Hartley, J. Chem. Soc., 633-642 (1938).

4) Rau, H., Photoisomerization of Azobenzenes in "Photoreactive Organic Thin Films," Ed. by Z. Sekkat and W. Knoll, Academic Press (2002) chapter 1, pp. 3-47.

5) L. M. Minsk, US Patent 2725377 (1955).

6) P. G. Egerton, E. Pitts and A. Reiser, Macromolecules, 14, 95-100 (1981).

7) K. Ichimura, Chem. Rev., 100, 1847-1873 (2000)

8) T. Seki, Polym. J., 36, 435-454 (2004).

9) T. Ikeda, J. Mater. Chem., 13, 2037-2057 (2003).

10) W. M. Gibbons, P. J. Shanon, S. T. Sun and B. J. Swetlin, Nature, 351, 49-51 (1991).

11) A. Natansohn and P. Rochon, Chem. Rev., 102, 4139-4176 (2002).

12) Sekkat, Z. and Knoll W. Ed., "Photoreactive Organic Thin Films,” Academic Press, San Diego, CA (2002).

13) T. Seki, Bull. Chem. Soc. Jan. (2007) in press.

14) T. Seki, Curr. Opin. Solid State Mater. Sci., 10, 241-248 (2007).

15) K. Ichimura, M. Momose, K. Kudo, H. Akiyama and N. Ishizuki, Langmuir, 11, 2341-2343 (1995).

16) T. Seki, K. Fukuda and K. Ichimura, Langmuir, 15, 5098-5101 (1999).

17) K. Ichimura, Y. Suzuki, T. Seki, A. Hosoki and K. Aoki, Langmuir, 4, 1214-1246 (1988).

18) T. Seki, M. Sakuragi, Y. Kawanishi, T. Tamaki, R. Fukuda and K. Ichimura, Langmuir, 9, 211-218 (1993).

19) G. Q. Lu, X. S. Zhao, Ed., "Nanoporous Materials: Science and Engineering," Imperial College Press, Vol. 4 (2004).

20) Kresge, C. T., Leonowicz, M. E., Roth, W. J., Vartuli, J. C. and Beck, J. S., Nature, 359, 710-712 (1992).
21) J. S. Beck, J. C. Vartuli, W. J. Roth, M. E. Leonowicz, C. T. Kresge, K. D. Schmitt, C. T-W. Chu, D. H. Olson, E. W. Sheppard, S. B. McCullen, J. B. Higgins and J. L. Schlenker, J. Am. Chem. Soc., 114, 10834-10843 (1992).

22) T. Yanagisawa, T. Shimizu, K. Kuroda and C. Kato, Bull. Chem. Soc. Jpn., 63, 988-992 (1990).

23) S. Inagaki, Y. Fukushima and K. Kuroda, J. Chem. Soc., Chem. Commun., 680-681 (1993).

24) A. Stein, B. J. Melde and R. C. Schroden, Adv. Mater., 12, 1403-1419 (2000).

25) B. J. Scott, G. Wirnsberger and G. D. Stucky, Chem. Mater., 13, 3140-3150 (2001)

26) F. Marlow, M. D. McGehee, D. Zhao, B. F. Chmelka and G. D. Stucky, Adv. Mater., 11, 632-636 (1999).

27) K. Kageyama, J. Tamazawa and T. Aida, Science, 285, 2113-2115 (1999).

28) A. Fukuoka, Y. Sakamoto, S. Guan, S. Inagaki, N. Sugimoto, Y. Fukushima, K. Hirahara, S. Iijima and M. Ichikawa, J. Am. Chem. Soc., 123, 3373-3374 (2001).

29) C.-M. Yang, H.-S. Sheu and K.-J. Chao, Adv. Funct. Mater., 12, 143-148 (2002).

30) H. W. Hillhouse, T. Okubo, J. W. Egmond and M. Tsapatsis, Chem. Mater., 9, 1505-1507 (1997).

31) M. Trau, N. Yao, E. Kim, Y. Xia, G. M. Whitesides and I. A. Aksay, Nature, 390, 674-676 (1997).

32) M. Ogawa, J. Am. Chem. Soc., 116, 7941-7942 (1994).

33) H. Yang, A. Kuperman, N. Coombs, S. Mamiche-Afara and G. A. Ozin, Nature, 379, 703-705 (1996).

34) I. A. Aksay, M. Trau, S. Manne, I. Honma, N. Yao, L. Zhou, P. Fenter, P. M. Eisenberger and S. M. Gruner, Science, 273, 892-898 (1996).

35) H. Miyata and K. Kuroda, Chem. Mater., 12, 49-54 (2000).

36) H. Miyata and K. Kuroda, Adv. Mater., 11, 1448-1452 (1999).

37) Y. Kawashima, M. Nakagawa, T. Seki and K. Ichimura, Chem. Mater., 14, 2842-2844 (2002).

38) Y. Kawashima, M. Nakagawa, K. Ichimura and T. Seki, $J$. Mater. Chem., 14, 328-335 (2004).

39) H. Fukumoto, S. Nagano, N. Kawatsuki and T. Seki, $A d v$. Mater., 17, 1035-1039 (2005).

40) H. Fukumoto, S. Nagano, N. Kawatsuki and T. Seki, Colloids Surf. A, 284, 309-314 (2006).

41) H. Fukumoto, S. Nagano, N. Kawatsuki and T. Seki, Chem. Mater., 18, 1226-1234 (2006).

42) N. Kawatsuki, T. Kawakami and T. Yamamoto, Adv. Mater., 13, 1337-1339 (2001).

43) N. Kawatsuki, K. Goto, T. Kawakami and T. Yamamoto, Macromolecules, 35, 706-713 (2002).

44) M. Schadt, H. Seiberle and A. Schuster, Nature, 381, 212-215 (1996).

45) M. Schadt, K. Schmitt, V. Kozinkov and V. Chingrinov, Jpn. J. Appl. Phys., 31, 2155-2164 (1992).

46) A. Hozumi, Y. Yokogawa, T. Kameyama, K. Hiraku, H. Sugimura, O. Takai and M. Okido, Adv. Mater., 12, 985-987 (2000).

47) H. Fukumoto, S. Nagano and T. Seki, Chem. Lett., 35, 180-181 (2006).

48) H. G. Zhu, D. J. Jones, J. Zajac, J. Roziere and R. Dutartre, Chem. Commun., 2568-2569 (2001).

49) J. E. Lydon, Curr. Opin. Colloid Interface Sci., 8, 480-490 (2004).

50) G. J. T. Tiddy, D. L. Mateer, A. P. Ormerod, W. J. Harrison and D. J. Edwards, Langmuir, 11, 390-393 (1995).

51) C. Ruslim, D. Matsunaga, M. Hashimoto, T. Tamaki and K. Ichimura, Langmuir, 19, 3686-3691 (2003).

52) C. Ruslim, M. Hashimoto, D. Matsunaga, T. Tamaki and K. Ichimura, Langmuir, 20, 95-100 (2004).

53) K. Ichimura, T. Fujiwara, M. Momose and D. Matsunaga, $J$. Mater. Chem., 12, 3380-3386 (2002). 
54) T. Fujiwara and K. Ichimura, J. Mater. Chem., 12, 3387-3391 (2002).

55) D. Matsunaga, T. Tamaki, H. Akiyama and K. Ichimura, Adv. Mater., 14, 1477-1480 (2002).

56) S.-W. Tam-Chang, W. Seo, I. K. Iverson and S. M. Casey, Angew. Chem. Int. Ed., 42, 897-900 (2003).

57) T. Schneider and O. D. Lavrentovich, Langmuir, 16, 52275230 (2000).

58) M. Hara, N. Mizoshita, S. Nagano and T. Seki, Langmuir, 23, 12350-12355 (2007)
59) T. Schneider, K. Artyushkova, J. E. Fulghum, L. Broadwater, A. Smith and O. D. Lavrentovich, Langmuir, 21, 2300-2307 (2005).

60) T. Yokoi, H. Yoshitake and T. Tatsumi, Chem. Mater., 15, 4536-4538 (2003).

61) S. Che, A. E. Garcia-Bennett, T. Yokoi, K. Sakamoto, H. Kunieda, O. Terasaki and T. Tatsumi, Nature Mater., 2, 801-805 (2003).

62) M. Hara, S. Nagano, N. Kawatsuki and T. Seki, submitted for publication. 